\title{
Dehydration of forward osmosis membrane in treating high salinity wastewaters: performances and implications
}

\author{
Baolong Zhao ${ }^{1}$, Mengxi Zhang ${ }^{1,2}$, Zhouwei Wang ${ }^{1}$, Jianfeng Song ${ }^{1,2}$, Myoung Jun Park ${ }^{4}$, Ho \\ Kyong Shon ${ }^{4}$, Xue-Mei $\mathrm{Li}^{1 *}$, Tao $\mathrm{He}^{1,3^{*}}$ \\ ${ }^{1}$ Laboratory for Membrane Materials and Separation Technology, Shanghai Advanced Research \\ Institute, Chinese Academy of Sciences, Shanghai 201203, China \\ ${ }^{2}$ University of Chinese Academy of Sciences, Beijing, 100049, China \\ ${ }^{3}$ School of Physical Science and Technology, ShanghaiTech University, Shanghai, 201210, \\ China \\ ${ }^{4}$ Centre for Technology in Water and Wastewater, School of Civil and Environmental \\ Engineering, University of Technology, Sydney (UTS), P.O. Box 123, 15 Broadway, NSW 2007, \\ Australia
}




\section{ABSTRACT}

Forward osmosis (FO) has shown advantages for desalinating high saline wastewater. Dehydration of FO membrane was demonstrated for both commercial cellulose triacetate and tailor-made thin-film composite membranes. A significant loss of FO flux was observed after membrane dehydration, but the pure water permeability and rejection properties remained about the same. Visual observation of the membrane dehydration was illustrated by appearance of opaque/white spots on the initially homogeneous membrane. It was shown that when the active skin layer was in contact with the saline solution before the support layer was brought into contact with an aqueous solution, membrane dehydration took place. The dehydration was ascribed to osmosis gradient across the active layer that caused water flow from membrane support layer to the active layer, resulting in support dehydration. The dehydrated membrane could be rewetted again by RO, illustrating that membrane dehydration is reversible. However, once dehydrated, a membrane with a much lower water flux will be resulting in.

\section{KEYWORDS}

Forward osmosis; dehydration; desalination; hollow fiber; thin-film composite; reverse osmosis 


\section{Introduction}

Forward osmosis (FO) is an osmotically driven membrane process without utilization of external hydraulic pressure ${ }^{1}$. FO has been experimentally proven as an intrinsically low fouling process $^{1-4}$. However, thermodynamically, FO would be more energy intensive than conventional reverse osmosis $(\mathrm{RO})$, when the recovery of the draw solution is required ${ }^{4}$. Therefore, the FO process may find applications mainly in special areas where either RO is not suitable due to high fouling tendency, high salinity, or the diluted draw solution can be directly used. For example, osmotic dilution (OD) of the shale gas drilling mud $^{5}$ and fertilizers ${ }^{6,7}$, and concentration of fruit juice using $\operatorname{sugar}^{8,9}$ are the most prosperous areas where FO is highly competitive to other membrane processes.

In OD of the produced water from oil/gas exploitation, the feed is highly saline and turbid, with TDS up to $70,000 \mathrm{mg} / \mathrm{L}$ or higher ${ }^{4,5,10,11}$. When treating such water, a draw solution of even higher salinity is needed. Therefore, the FO membrane is under a constant stress caused by osmotic pressure. Frequently, the membrane flux is much lower than expected because of the severe internal concentration polarization (ICP) ${ }^{1,12-15}$, membrane fouling and/or scaling ${ }^{3,16,17}$. Optimization of the membrane support structure could effectively reduce the $\mathrm{ICP}^{12,14,15,18-23}$. By modification of membrane surface, decrease in membrane fouling or scaling was also reported ${ }^{24}$. Pre-treatment was used to reduce the fouling propensity in FO process ${ }^{10}$. However, when treating high salinity water streams, one of the often disregarded factors causing low FO water flux is the FO membrane dehydration.

Membrane dehydration often leads to a significant loss of FO flux along with the FO process. The dehydration can also be visualized by the appearance of white spots on the membrane ${ }_{3}$ 
surface. However, so far, the origin of membrane dehydration is not clearly understood, and there has not been a systematic investigation on the membrane dehydration. Characteristics of dehydrated membranes are not clearly addressed in literature, neither membrane performance recovery after dehydration. This paper presents the first investigation on the FO membrane dehydration especially for treating high salinity water. Effects of membrane materials and operation mode on the dehydration are explored. The performance of the FO membrane after dehydration is evaluated and a mechanism for the dehydration of FO membrane is proposed. The solutions to avoid dehydration and performance recovery of dehydrated FO membrane are discussed, which will contribute to some guidelines for the application of FO in treating high salinity waters.

\section{Experimental}

\subsection{Materials and chemicals}

Polysulfone (PSf P-3500, Solvay) was dried at $100^{\circ} \mathrm{C}$ for at least one week before use. Analytical grade (AR) N,N-dimethylacetamide (DMAc), polyethylene glycol (PEG400), n-hexane, alcohol, N-methyl-2-pyrrolidone (NMP) n-hexane, and sodium chloride ( $\mathrm{NaCl}$ ) were provided by Sinopharm Groups. 1,2-Phenylenediamine (MPD, 99\%) and trimesoyl chloride (TMC, 98\%) were purchased from Sigma-Aldrich. Polyethersulfone (PES) hollow fiber membranes were supplied by Nanjing Altrateck Co. Ltd. Underground brine (UGB) was supplied by Shangdong Haihua Chemical Industry. Commercial cellulose triacetate (CTA) membrane was supplied by Hydration Technology Innovations (HTI).

\subsection{Preparation and characterization of the TFC membranes}




\subsubsection{PSf flat-sheet TFC membrane}

Flat-sheet TFC membrane was prepared by following a previously reported procedures ${ }^{23}$. PSf support membrane was initially made and PSf/PEG-400/DMAc (18/8/74 wt.\%) was mixed in a three-neck flask at $65^{\circ} \mathrm{C}$ overnight till a clear solution was obtained. The polymer solution was filtered and then de-gassed in an oven at $60^{\circ} \mathrm{C}$ overnight. The solution was cast on a dry glass plate using an automatic casting apparatus (Elcometer 4340, Elcometer Asia Pte. Ltd) pre-set with $150 \mu \mathrm{m}$ gap. The nascent film was then immersed into a water bath $\left(30{ }^{\circ} \mathrm{C}\right)$ for precipitation. Subsequently, PSf membranes were washed thoroughly and stored in DI water before further usage. A polyamide selective layer was formed on top surface of the PSf membrane via interfacial polymerization as follows. The wet PSf support was initially dried with an air knife and brought into contact with water phase for 2 min. The composition of water phase was adopted from the report of Kim et al ${ }^{25}$ at a $\mathrm{pH}$ of 11.2. The excessive aqueous solution was decanted and the membrane surface was blown dry under a stream of nitrogen gas. Afterwards, the membrane was brought into contact with the organic phase (TMC hexane solution, $0.15 \mathrm{wt}$ $\%$ ) for $1 \mathrm{~min}$. The membrane was then air-dried for $2 \mathrm{~min}$ and cured in an oven at $100{ }^{\circ} \mathrm{C}$ for 3 min. The fresh membranes were stored in DI water before further test.

\subsubsection{PES hollow fiber TFC membranes}

Preparation of hollow fiber (HF) TFC FO membranes followed the previously reported procedure $^{18-21,26}$. Ten pieces of PES HF membranes were potted in a nylon tube (with an effective fiber length of $25 \mathrm{~cm}$ and effective membrane surface area of $85 \mathrm{~cm}^{2}$ ). A fresh water phase as above was introduced to the bore side of the fibers with a resident time of 2 min before discharged. After blowing dry by nitrogen gas, organic phase was led to the bore for contact ${ }_{5}$ 
time of $1 \mathrm{~min}$. The membrane was cured with hot water $\left(85^{\circ} \mathrm{C}\right)$ for $5 \mathrm{~min}$. Finally, the membrane module was immersed in DI water before further characterization.

\subsubsection{Characterization of FO membranes}

The standard characterization protocol for flat-sheet FO membranes was adopted in this test ${ }^{27}$. The intrinsic pure water permeability (A) and solute permeability coefficient (B) of the membranes were determined using a cross-flow RO filtration system (Sterlitech Corporation). Detailed procedures are provided as Supplementary Information (S1 Preparation and characterization of forward osmosis membranes).

\subsection{Dehydration experiment}

The dehydration test is schematically shown in Fig.1. A flat-sheet FO membrane sample (40 $\mathrm{mm} \times 40 \mathrm{~mm}$ ) was glued to the pre-cut opening $(30 \mathrm{~mm} \times 30 \mathrm{~mm})$ in a polyethylene (PE) film (25 $\mu \mathrm{m}$ thickness). This ensures that only one side of the membrane was in contact with the liquid when being placed above a sample solution (Fig. 1). Dehydration of the membranes was then observed visually. For HF TFC FO membrane, the high salinity solution was fed to the bore (flow rate of $0.66 \mathrm{~m} / \mathrm{s})$ or shell side $(0.25 \mathrm{~m} / \mathrm{s})$ of the membrane using a calibrated gear pump for a certain period of time. Afterwards, the membrane module was rinsed using 2 L DI water for 30 min for at least 3 times to completely rinse out any remaining salt. The cleaned HF membrane was then tested in the AL-FS mode, i.e. the membrane active layer facing the feed solution (Supplementary Information S1). 


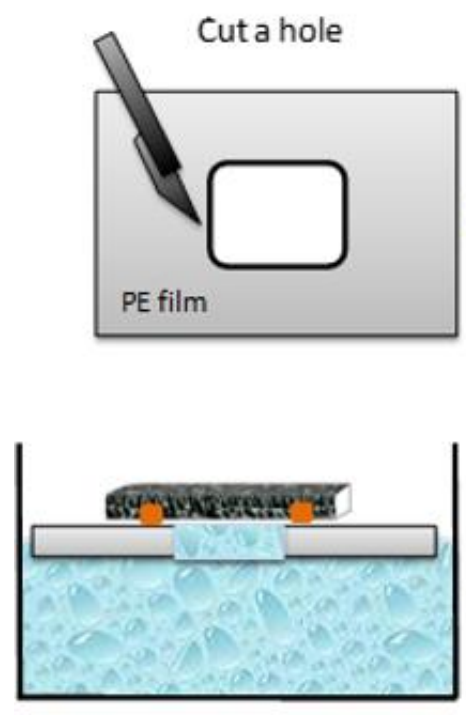

Put it on water surface

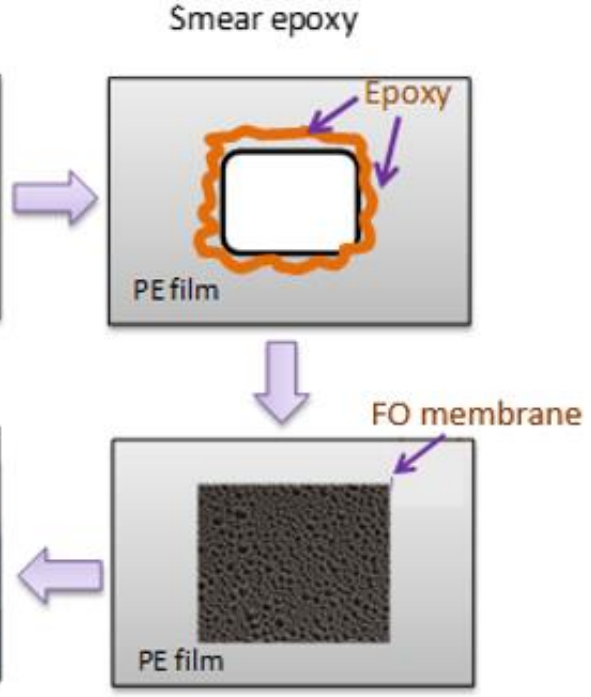

Glue FO membrane

Fig. 1 Schematic illustration of the sample preparation process for the dehydration test of flatsheet FO membranes.

\subsection{Analytical methods}

Water quality data including $\mathrm{pH}$, electric conductivity, turbidity, and total hardness were analyzed following standard methods ${ }^{8}$. Ion concentrations were determined by inductively coupled plasma-atomic emission spectroscopy (ICP-AES, ICPE-9000, Shimadzu, Kyoto, for cations) and ion chromatography (LC20AT, Shimadzu, Kyoto, for anions). As described in Table 1, the model high salinity brine (HSB) solution contains high concentrations of $\mathrm{Na}^{+}, \mathrm{Mg}^{2+} \mathrm{Ca}^{2+}$, chloride, and sulfate. Potassium, strontium and arsenic were also found with TDS of 120,347 $\mathrm{mg} / \mathrm{L}$. 
Table 1 Water quality data of the underground brine used in this study

\begin{tabular}{|c|c|}
\hline Analytes & Concentration \\
\hline Sodium (mg/L) & 13,968 \\
\hline Calcium (mg/L) & 7,230 \\
\hline Potassium (mg/L) & 1,862 \\
\hline Magnesium (mg/L) & 10,864 \\
\hline Strontium (mg/L) & 73 \\
\hline Arsenic $(\mathrm{mg} / \mathrm{L})$ & 71 \\
\hline Chloride (mg/L) & 62,137 \\
\hline Sulfate $(\mathrm{mg} / \mathrm{L})$ & 11,986 \\
\hline Bicarbonate (mg/L) & 247 \\
\hline TDS (mg/L) & 120,347 \\
\hline
\end{tabular}

\section{Results and discussion}

\subsection{Dehydration of FO membranes}


The high salinity underground brine (UGB) is a raw material for the production of $\mathrm{Na}_{2} \mathrm{CO}_{3}$. The production process involves evaporation of UGB, which requires a large area of land and is time-consuming. In order to minimize the footprint of the production process and improve the process efficiency, we have proposed the use of FO to concentrate the brine. Because of the high salinity of the UGB (Table 1), saturated $\mathrm{NaCl}$ draw solutions were used for the FO concentration of the UGB.

Both CTA and tailor-made TFC FO membranes were tested, and the water fluxes are shown in Fig. 2. Two scenarios have been found for both membranes. For example, an initial water flux of $9 \mathrm{~L} / \mathrm{m}^{2} \mathrm{~h}$ was found for the CTA membrane, which was $11 \mathrm{~L} / \mathrm{m}^{2} \mathrm{~h}$ for the TFC membrane. Gradual decline of the water fluxes for both CTA and TFC membranes were observed, which was expected because of the concentration of feed streams. However, occasionally, we have found much lowered water fluxes, about $90 \%$ lower than expected, for both membranes, which was ascribed to membrane dehydration. This was evidenced by the appearance of white spots in both FO membranes (Figs. 2B and 2C) after FO concentration processes.

Based on above observation, the membrane dehydration is characterized by much lowered water flux due to loss of effective diffusion area. But it remained unclear what are the governing factors for the membranes dehydration to take place. 

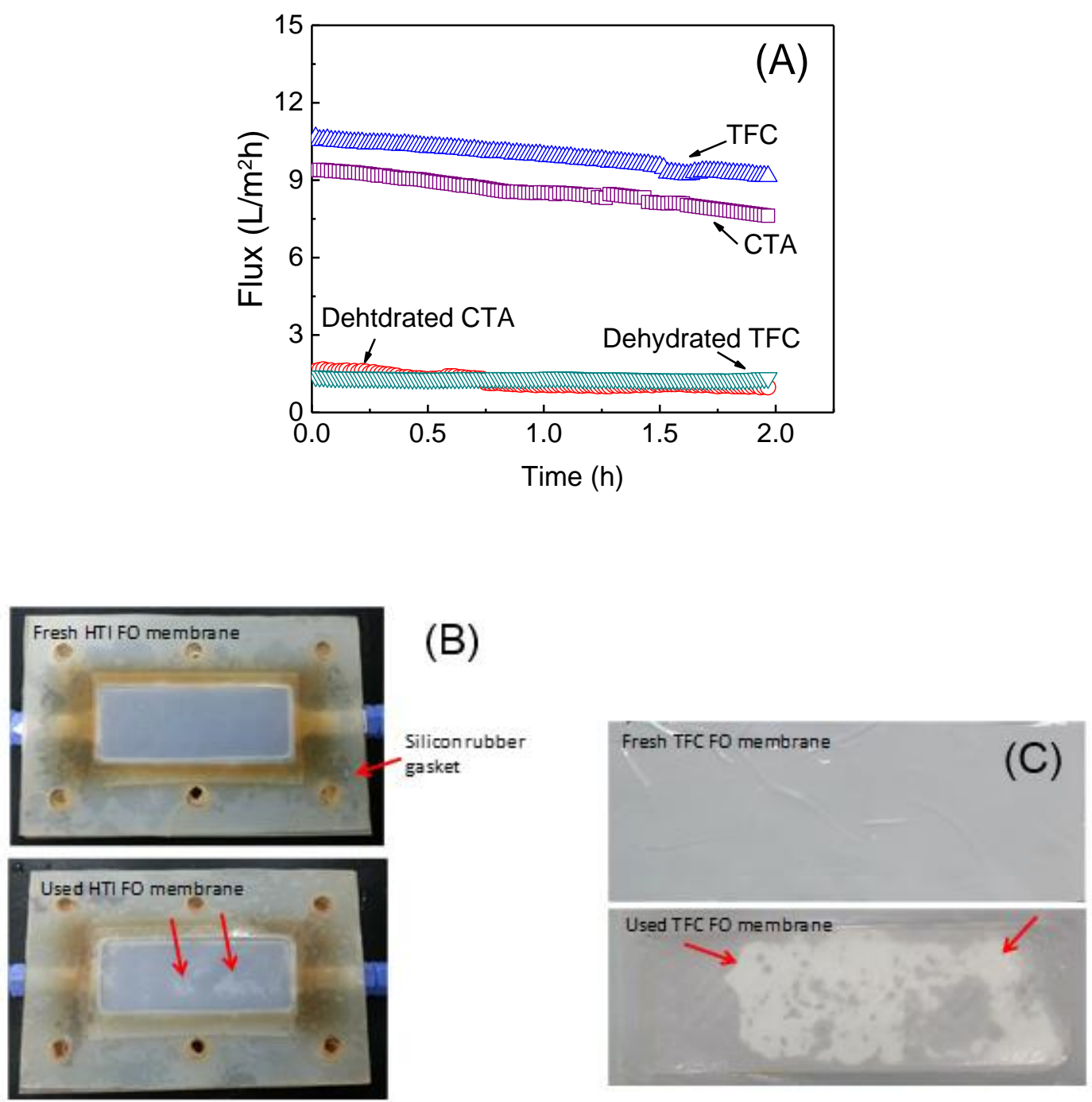

Fig. 2 The FO fluxes of the CTA and TFC FO membranes using high salinity brine as the feed and saturated $\mathrm{NaCl}$ solution as the draw solution (A) operated in the AL-FS mode. Experiments were conducted continuously for 2 hours at cross flow velocity of $8.8 \mathrm{~cm} / \mathrm{s}$ at $25 \pm 2{ }^{\circ} \mathrm{C}$. Photo images of the pristine and used HTI CTA membrane (B). Photo images of the pristine and used tailor-made TFC membrane (C). Arrows are pointing at the dehydrated area. The membranes were rinsed with DI water after usage. 


\subsection{Controlled dehydration of CTA and TFC membranes}

In order to understand the membrane dehydration, a controlled dehydration process was designed. As shown schematically in Fig. 1, a precut membrane coupon was glued onto a piece of PE film, which allowed the membrane to float above the aqueous streams. Moreover, only one side (active layer facing the feed or support layer facing the feed solution) of the membrane may be in contact with the feed at a time. The saline feed solution with composition listed in Table 1 was used. Fig. 3 shows the photo images of both CTA and TFC FO membranes after in contact with the saline solution. When the active layer contacted the solution, dehydration of the membrane was observed as indicated by the appearance of white spots, which grew larger with time in both membranes (Fig 3A). When the support layer was in direct contact with the solution, both membranes remained in wet state (Fig 3B). This observation suggests that, regardless of the membrane material, dehydration is more affected by the orientation of the membrane with reference to which of its side is in contact with the high salinity water and the supply of the other solution.
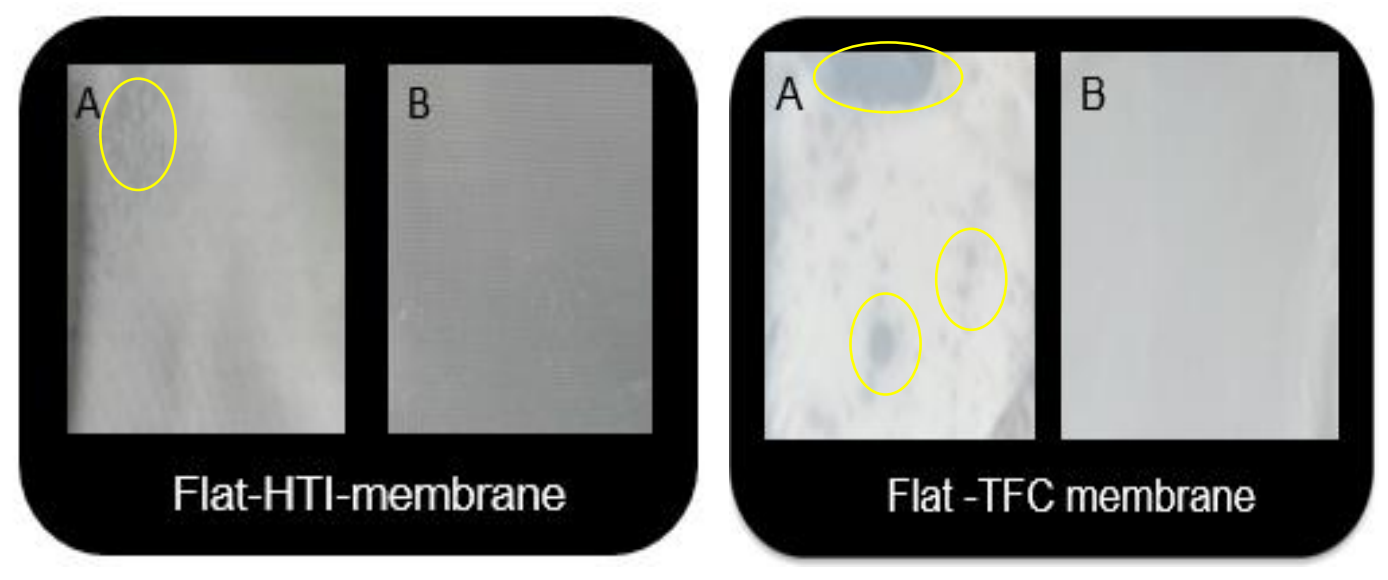
Fig. 3 Photo images of flat-sheet HTI CTA and tailor-made TFC FO membranes after contacting the high saline solutions for 60 seconds. (A) active skin layer facing the saline solution; (B) support layer facing the saline solution. The circles highlight the wet spots remaining in the membrane. CTA FO membrane represents a hydrophilic structure and TFC membrane represents a FO membrane with hydrophobic support.

\subsection{Effect of dehydration on the FO and RO performance}

In order to verify the membrane orientation effects on membrane performance, bench scale HF TFC membranes were prepared for this experiment (Supplementary Information Fig. S1 and Table S1). When the HF membrane was used, there was no external mechanical stress to damage the membrane: thus preventing problems encountered in flat-sheet FO membranes, where damages by the gasket are frequently observed. Three tests (same solution as listed in Table 1) were performed: (i) Support layer (shell side) contacted the saline solution first, (ii) Active surface layer (bore side) contacted the saline solution first, and (iii) Active surface (bore) and support side (shell) contacted the saline solution simultaneously.

The time lag between the supply of the two solutions across the membrane was controlled. The time lag effects on the membrane flux, rejection, and pure water permeability (experimental details are described in Supplementary Information S1.2) were characterized as shown in Fig 4. The FO fluxes of the membranes showed different scenarios: (1) when the support layer was first in contact with the saline solution, the membrane FO flux remained the same (not shown here); similar to the case when both the active and support layers contacted simultaneously the saline solutions (0s time lag). However, when the active layer was in contact firstly with the saline solution, the FO flux decreased to $50 \%$ for $40 \mathrm{~s}$ time lag, with no further decline after longer 
time lag (120 s). It probably means that the FO membrane occurred nearly instantaneously; reaching a maximum extent within a very short time. On the other hand, both the pure water permeability (A value) and the rejection of membranes were about nearly the same (Fig. 4B). These results appear to confirm that the membrane active layer remained intact even when the membrane was dehydrated. Moreover, it appeared that the RO operation was able to hydrate the dehydrated membrane.
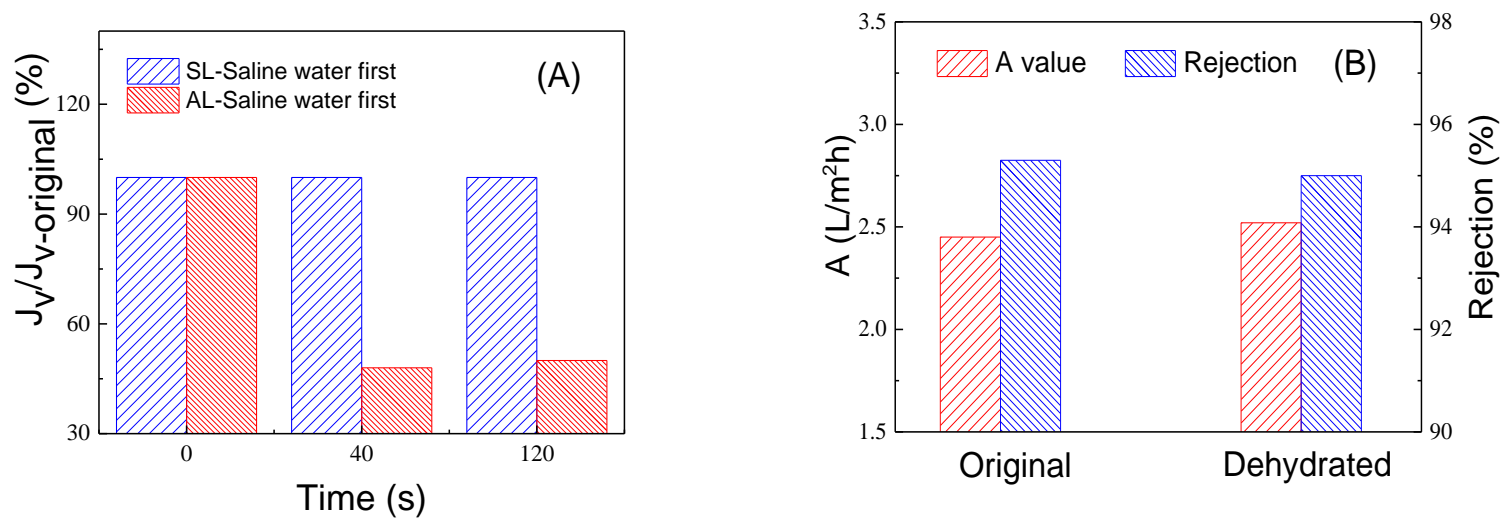

Fig. 4 (A) Effects of different time interval supplying the saline solution to between the active layer (AL) and the support layer (SL) on the FO flux. The Y axis is presented as the ratio of the FO flux to the original flux. The FO test was carried out at AL-FS mode using $0.5 \mathrm{M} \mathrm{NaCl}$ solution as the draw solution and DI water as the feed at $25{ }^{\circ} \mathrm{C}$. Before the $\mathrm{FO}$ test, the membranes were thoroughly cleaned with DI water to remove any salt left in the membrane structure. (B) The water permeability and rejection of the hollow fiber TFC membranes before and after dehydration. A value was determined using pure water as feed. The test conditions of RO: operation pressure $=2$ bar, feed solution $=100 \mathrm{mg} / \mathrm{L} \mathrm{NaCl}$ solution at $25{ }^{\circ} \mathrm{C}$.

\subsection{Proposed mechanism of dehydration}


Based on the above observation, a dehydration mechanism is proposed as illustrated in Fig. 5. When the support layer is in contact with the saline water the salt diffuses into the support layer and is mixed with water. The net salt diffusion ends when the chemical potential of the water inside the porous structure and the bulk liquid phase reaches osmotic equilibrium. However, when the saline water is in contact with the semipermeable active skin layer, an osmotic pressure gradient is built up between water in the porous structure and the saline water. As a result, water molecules in the porous structure diffuse across the active layer into the saline water driven by the osmotic pressure gradient, leading to a continuous dehydration of the support layer.

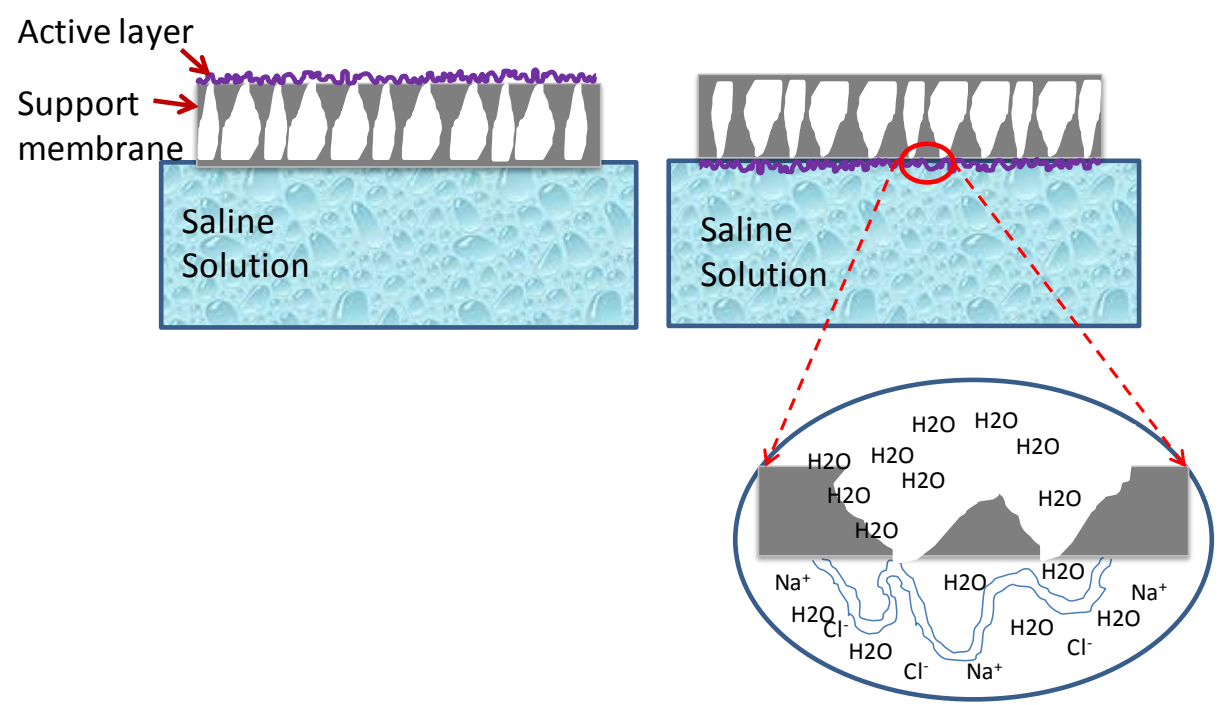

Fig. 5 Schematic illustration of proposed dehydration mechanism of TFC FO membrane in contact with a saline solution with very high osmotic pressure. (A) the support layer facing the saline solution; (B) the active layer facing the saline solution. The snapshot shows water molecule diffusion through the active layer.

Since water is a non-compressible phase, the loss of water through the skin layer is continuously compensated by the water diffused from the upper part in the porous structure. 
The accurate starting point of dehydration is unknown, but most probably from the far side of the support layer to the active skin. Based on this mechanism, the dehydration process is independent of membrane materials for both the active layer and support layer. Therefore, both HTI CTA and TFC (PSf support and PES support) membranes showed the same dehydration phenomenon (Fig. 2 and 3). The osmotic pressure of the saline solution gives impact on the rate of dehydration. At a lower osmotic pressure, the process for dehydration is slower; when $0.5 \mathrm{M}$ $\mathrm{NaCl}$ solution was utilized as a test saline solution for both CTA and TFC FO membranes, dehydration was also observed at a slower rate when the active layer was in direct contact with the saline solution. Moreover, because dehydration occurred mostly in the support layer, pure water permeability and rejection properties remain the same, as confirmed by the RO test.

\section{Conclusions}

The dehydration of FO membrane has been investigated using commercial CTA and home-made TFC membranes. Results showed that membrane dehydration is characterized by the loss of FO flux up to $50 \%$, but the A value and rejection properties remain unchanged. Visual observation of the membrane dehydration is demonstrated by the appearance of opaque or white spots on the membrane surface. Careful designed experiments illustrated that the membrane dehydration was caused by an osmotic pressure gradient that draws water flow from the support to the active layer and lack of water supply in the membrane support. Especially for the treatment of high salinity waste streams, the membrane dehydration may become serious because the dehydration occurs within a short time of less than $1 \mathrm{~min}$. We have shown that RO could be used to rehydrate the FO membranes. However, sufficient water supply in the support layer is 
critical to avoid membrane dehydrations. Results reported here have provided some insights on membrane dehydration mechanism, which will be critical for FO applications where high salinity feed streams are concerned.

\section{AUTHOR INFORMATION}

\section{Corresponding Author}

*Phone: 0086-21-20325162, Fax: 0086-21-20325034, email: lixm@sari.ac.cn, het@sari.ac.cn.

\section{Notes}

The authors declare no competing financial interest.

\section{ACKNOWLEDGMENT}

We acknowledge the financial support from Natural Science Foundation of China (21176119),

National Key Basic Research Program (973 Program, No. 2009CB623402, 2012CB932800), TMSR from Chinese Academy of Sciences (Project No. XDA02020100) and ARC Future Fellowship.

\section{REFERENCES}

1. Cath, T.; Childress, A.; Elimelech, M., Forward osmosis: Principles, applications, and recent developments. Journal of Membrane Science 2006, 281, (1-2), 70-87.

2. Chung, T.-S.; Zhang, S.; Wang, K. Y.; Su, J.; Ling, M. M., Forward osmosis processes: Yesterday, today and tomorrow. Desalination 2012, 287, 78-81.

3. Mi, B.; Elimelech, M., Organic fouling of forward osmosis membranes: Fouling 
reversibility and cleaning without chemical reagents. Journal of Membrane Science 2010, 348, $(1-2), 337-345$.

4. Shaffer, D. L.; Werber, J. R.; Jaramillo, H.; Lin, S.; Elimelech, M., Forward osmosis: Where are we now? Desalination 356, (0), 271-284.

5. Hickenbottom, K. L.; Hancock, N. T.; Hutchings, N. R.; Appleton, E. W.; Beaudry, E. G.; $\mathrm{Xu}, \mathrm{P} . ;$ Cath, T. Y., Forward osmosis treatment of drilling mud and fracturing wastewater from oil and gas operations. Desalination 2013, 312, 60-66.

6. Phuntsho, S.; Shon, H. K.; Hong, S.; Lee, S.; Vigneswaran, S., A novel low energy fertilizer driven forward osmosis desalination for direct fertigation: Evaluating the performance of fertilizer draw solutions. Journal of Membrane Science 2011, 375, (1-2), 172-181.

7. Phuntsho, S.; Shon, H. K.; Majeed, T.; El Saliby, I.; Vigneswaran, S.; Kandasamy, J.; Hong, S.; Lee, S., Blended Fertilizers as Draw Solutions for Fertilizer-Drawn Forward Osmosis Desalination. Environmental Science \& Technology 2012, 46, (8), 4567-4575.

8. Clesceri, L. S., Greenberg, A.E. \& Eaton, A.D. (eds), Standard Methods for the Examination of Water and Wastewater, 20th edn. American Public Health Association/American Water Works Association/Water Environment Federation: Washington, DC, USA, 1999.

9. Garcia-Castello, E. M.; McCutcheon, J. R.; Elimelech, M., Performance evaluation of sucrose concentration using forward osmosis. Journal of Membrane Science 2009, 338, (1-2), 61-66.

10. Li, X.-M.; Zhao, B.; Wang, Z.; Xie, M.; Song, J.; Nghiem, L. D.; He, T.; Yang, C.; Li, C.; Chen, G., Water reclamation from shale gas drilling flow-back fluid using a novel forward osmosis-vacuum membrane distillation hybrid system. Water Science Technology 2014, 69, (5), 1036-1044. 
11. Shaffer, D. L.; Arias Chavez, L. H.; Ben-Sasson, M.; Romero-Vargas Castrillon, S.; Yip, N. Y.; Elimelech, M., Desalination and reuse of high-salinity shale gas produced water: drivers, technologies, and future directions. Environ Sci Technol 2013, 47, (17), 9569-83.

12. Gray, G. T.; McCutcheon, J. R.; Elimelech, M., Internal concentration polarization in forward osmosis: role of membrane orientation. Desalination 2006, 197, (1-3), 1-8.

13. Park, M.; Lee, J. J.; Lee, S.; Kim, J. H., Determination of a constant membrane structure parameter in forward osmosis processes. Journal of Membrane Science 2011, 375, (1-2), 241-248.

14. Tang, C. Y.; She, Q.; Lay, W. C. L.; Wang, R.; Fane, A. G., Coupled effects of internal concentration polarization and fouling on flux behavior of forward osmosis membranes during humic acid filtration. Journal of Membrane Science 2010, 354, (1-2), 123-133.

15. Wang, K. Y.; Ong, R. C.; Chung, T.-S., Double-Skinned Forward Osmosis Membranes for Reducing Internal Concentration Polarization within the Porous Sublayer. Industrial and Engineering Chemistry Research 2010, 49, (10), 4824-4831.

16. Liu, P.; Gao, B.; Shon, H. K.; Ma, D.; Rong, H.; Zhao, P.; Zhao, S.; Yue, Q.; Li, Q., Water flux behavior of blended solutions of ammonium bicarbonate mixed with eight salts respectively as draw solutions in forward osmosis. Desalination 2014, 353, (0), 39-47.

17. Xie, M.; Nghiem, L. D.; Price, W. E.; Elimelech, M., Impact of humic acid fouling on membrane performance and transport of pharmaceutically active compounds in forward osmosis. Water Research 2013, 47, (13), 4567-4575.

18. Li, G.; Wang, Z.; Li, C.; Li, X.-M.; He, T.; Gao, C., Preparation and characterization of hollow fiber forward osmosis membrane by interfacial polymerization. CIESC Journal 2014, 65, (8), 3082-3088. 
19. Setiawan, L.; Wang, R.; Li, K.; Fane, A. G., Fabrication of novel poly(amide-imide) forward osmosis hollow fiber membranes with a positively charged nanofiltration-like selective layer. Journal of Membrane Science 2011, 369, (1-2), 196-205.

20. Setiawan, L.; Wang, R.; Li, K.; Fane, A. G., Fabrication and characterization of forward osmosis hollow fiber membranes with antifouling NF-like selective layer. Journal of Membrane Science 2012, 394-395, (0), 80-88.

21. Shi, L.; Chou, S. R.; Wang, R.; Fang, W. X.; Tang, C. Y.; Fane, A. G., Effect of substrate structure on the performance of thin-film composite forward osmosis hollow fiber membranes. Journal of Membrane Science 2011, 382, (1-2), 116-123.

22. Wang, K. Y.; Chung, T.-S.; Amy, G., Developing thin-film-composite forward osmosis membranes on the PES/SPSf substrate through interfacial polymerization. AIChE Journal 2012, $58,(3), 770-781$.

23. Xiao, P.; L, N.; Yin, Y.; Li, X.-M.; Zhang, M.; Chen, G.; Song, J.; He, T., A Sacrificial-layer Approach to Fabricate Polysulfone Support for Forward Osmosis Thin-film Composite Membranes with Reduced Internal Concentration Polarisation. Journal of Membrane Science 2015, 481, 106-114.

24. Lu, X. L.; Castrillon, S. R.-V.; Shaffer, D. L.; Ma, J.; Elimelech, M., In situ surface chemical modification of thin-film composite forward osmosis membranes for enhanced organic fouling resistance. Environmental Science \& Technology 2013, 47, (21), 12219-12228.

25. Kim, S. H., Kwak, S.Y., Suzuki, T., Positron annihilation spectroscopic evidence to demonstrate the flux-enhancement mechanism in morphology-controlled thin-film-composite (TFC) membrane. Environmental Science \& Technology 2005, 39, (6), 1764-1770.

26. Wang, R.; Shi, L.; Tang, C. Y.; Chou, S.; Qiu, C.; Fane, A. G., Characterization of novel 
forward osmosis hollow fiber membranes. Journal of Membrane Science 2010, 355, (1-2), 158-167.

27. Cath, T. Y.; Elimelech, M.; McCutcheon, J. R.; McGinnis, R. L.; Achilli, A.; Anastasio, D.;

Brady, A. R.; Childress, A. E.; Farr, I. V.; Hancock, N. T.; Lampi, J.; Nghiem, L. D.; Xie, M.;

Yip, N. Y., Standard Methodology for Evaluating Membrane Performance in Osmotically

Driven Membrane Processes. Desalination 2013, 312, 31-38. 